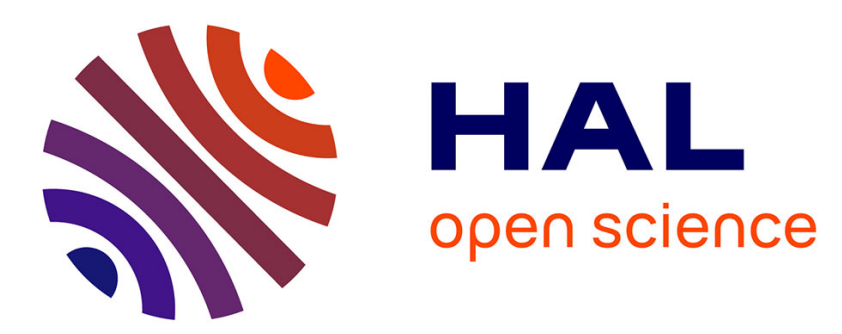

\title{
Colloidal particle resuspension: on the need for refined characterisation of surface roughness
}

Christophe Henry, Jean-Pierre Minier

\section{To cite this version:}

Christophe Henry, Jean-Pierre Minier. Colloidal particle resuspension: on the need for refined characterisation of surface roughness. Journal of Aerosol Science, 2018, 118, pp.1-13. 10.1016/j.jaerosci.2018.01.005 . hal-02054795

\section{HAL Id: hal-02054795 \\ https://hal.science/hal-02054795}

Submitted on 2 Mar 2019

HAL is a multi-disciplinary open access archive for the deposit and dissemination of scientific research documents, whether they are published or not. The documents may come from teaching and research institutions in France or abroad, or from public or private research centers.
L'archive ouverte pluridisciplinaire HAL, est destinée au dépôt et à la diffusion de documents scientifiques de niveau recherche, publiés ou non, émanant des établissements d'enseignement et de recherche français ou étrangers, des laboratoires publics ou privés. 


\title{
Colloidal particle resuspension: on the need for refined characterisation of surface roughness
}

\author{
Christophe Henry ${ }^{\mathrm{a}, \mathrm{b}, *}$, Jean-Pierre Minier ${ }^{\mathrm{c}}$ \\ ${ }^{a}$ Laboratoire Lagrange, Université de la Côte d'Azur, OCA, CNRS, 06304 Nice Cedex 4, France \\ ${ }^{b}$ Institute of Fluid-Flow Machinery, Polish Academy of Sciences, ul. Fiszera 14, 80-231 Gdańsk, Poland \\ ${ }^{c}$ EDF R\&D, Mécanique des Fluides, Energie et Environnement, 6 quai Watier, 78400 Chatou, France
}

\begin{abstract}
This paper highlights the role played by surface roughness on the resuspension of nanoand micro-sized particles and, in particular, the need to extract more information from measurements of the surface profile than typical values such as the average roughness $R_{a}$ and the rms roughness $R_{r m s}$ (usually obtained through AFM or SEM measures). For that purpose, standard experimental measurements of surface roughness are analysed. Then, numerical results obtained with a stochastic model for particle resuspension are analysed and compared to experimental data. This analysis reveals that particle resuspension can only be properly captured with more detailed representations of surface roughness that include information on the distribution of the curvature radius and surface coverage of roughness features.
\end{abstract}

Keywords: Reentrainment, resuspension, particle, adhesion, roughness, AFM

\section{Contents}

1 Introduction $\quad 2$

1.1 Particle resuspension phenomena . . . . . . . . . . . . . . 2

1.2 Purpose of the paper .......................... 4

2 Present modelling approach $\quad 5$

${ }^{*}$ Corresponding author

Email address: christophe.henry@mines-paris.org (Christophe Henry) 
2.1 Existing models for particle resuspension . . . . . . . . . . . . 5

2.2 Description of the present modelling approach . . . . . . . . . . . . 7

3 Measurement of surface roughness in the context of particle resuspension 9

3.1 Current approaches to measure roughness features . . . . . . . . . . 9

3.2 Selected case of colloid resuspension from rough substrates . . . . . . . . 12

4 Analysis of numerical results for particle resuspension $\quad 14$

4.1 Numerical parametrisation . . . . . . . . . . . . . . . 14

4.2 Numerical results . . . . . . . . . . . . . . . . . . 15

5 Discussion: towards refined descriptions of surface roughness 18

5.1 Insights into additional information needed . . . . . . . . . . . . . 19

5.2 A refined characterisation of surface roughness for particle resuspension . . 21

6 Conclusion $\quad 23$

\section{Introduction}

\subsection{Particle resuspension phenomena}

Particle resuspension is the process whereby particles deposited on a surface are detached from it and re-entrained into the fluid flow. It is an ubiquitous phenomena which has been studied extensively over the last decades. As emphasised in recent reviews $[3,11,14,34]$, it has received renewed attention over recent years due to its role in various environments, for example in sediment dynamics [6], for the issue of the re-entrainment of hazardous materials such as radioactive particles in nuclear power plant accidents [27] or in the context of walking-induced resuspension of airborne particles in hospitals [23].

One of the main challenges related to particle resuspension comes from its multidisciplinary aspects, which involves the coupling between fluid dynamics (particle-fluid interactions), interface chemistry (particle-surface adhesion forces) and material physics (surface 
roughness). Indeed, particle resuspension results from the competition between two opposite forces/torques: on the one hand, hydrodynamical forces that tend to pull or drag particles along the wall and, on the other hand, adhesion forces that tend to maintain these deposited particles on the wall $[2,19,22]$.

Hydrodynamical effects comprise lift and drag forces which play a different role depending on the mechanisms at play in particle dynamics. It is however important to note that these hydrodynamical forces are relatively well understood. Consequently, what is mostly at stake in particle resuspension modelling is to capture the distribution of adhesion forces. These forces result from the interaction between surfaces and thus depend on a number of factors including those related to the nature of the surfaces in contact and to their geometrical characteristics (such as surface roughness). At this point, it is worth mentioning that adhesion forces differs if single particles are deposited on a rough substrate or if particles form multilayered deposits. The resuspension of multilayered deposits adds indeed further complexity since adhesion/cohesion forces depend on the morphology of the deposit formed. In the present article, we focus on single particles resuspended from rough surfaces and the case of multilayered resuspension is left out for future studies. For single particles attached to the surface, several recent measurements of surface forces have shown that adhesion forces are significantly affected by the irregular nature of surfaces $[1,9,10,24,25,30,32,33]$. These random variations of surface features met by deposited particles imply that adhesion forces can take a range of values and that the corresponding distribution needs to be characterised statistically either through its CDF (Cumulative Distribution Function) or its PDF (Probability Density Function).

One of the key challenge in particle resuspension is that it spans over a very long timescale. In the context of radioactive or noxious particles, resuspension is usually decomposed in two parts (see for instance $[13,4,26]$ ): an initial short-term resuspension and a longterm resuspension. Short-term resuspension is characterised by the removal of particles that are weakly bounded to the surface, i.e. with small adhesion forces, whereas longterm resuspension involves the removal of particles that strongly adhere to the surface. The different behaviours between short-term and long-term resuspension rates is thus a 
direct consequence of the broad distribution in adhesion forces and, to a lesser extent, in hydrodynamic forces. As a result, when trying to ensure that the fraction of resuspended particles remains always below a given threshold value as in the case of nuclear safety (e.g. in $[4,28]$ ), detailed characterisations of the distribution of adhesion forces must be available. In particular, this includes information not only on the first few moments but also on the tails of these distributions (where extreme events occur).

\subsection{Purpose of the paper}

Drawing on these arguments, we have developed a model for particle resuspension that includes fine calculations of the adhesion forces between particles and rough surfaces (see [16]). This allows us to obtain the distribution of adhesion forces as an outcome of the modelling approach. The present approach can thus provide refined information for more macroscopic models, such as classical kinetic models (where the adhesion force distribution is an input [26]), as well as to design new empirical formulae for the resuspension rate/adhesion force distribution that remain valid for a wide range of cases. This is further motivated by the fact that previous numerical results have been shown to compare well with experimental data on adhesion force and to reproduce various forms of the adhesion force distribution (including two-peaks, Gaussian or log-normal distributions) [16]. Besides, this model for particle resuspension also allows us to extract the time-dependence of the resuspension rate that includes the effects of surface roughness. It has also been compared to existing experimental data on resuspension in previous papers [12, 16, 15]. Such information will help to design and check the validity of empirical formulas for the resuspension rate which are source terms in some models for indoor resuspension [21].

Yet, one of the key difficulties encountered when assessing the model lies in the fact the amount of information on the topography of surfaces used in resuspension experiments is limited. In a recent paper, Barth et al. [2] measured the resuspension of colloidal particles from rough substrates in a turbulent channel airflow at various friction velocities. These measurements provide not only information on the short-term resuspension (here after a 60 s exposure to a flow) but also on the roughness characteristics of the substrate. More 
precisely, surface roughness is quantified through a number of statistical numbers, such as the well-know $R_{a}$ value or similar quantities. It remains to be seen whether this is sufficient for particle resuspension studies.

The aim of the present paper is thus to investigate whether current characterisations of surface roughness are adequate to fully capture particle resuspension using the recent measurements of Barth et al. [2] for short-term resuspension. In that sense, the objectives of this paper are three-fold:

- to illustrate how surface roughness affects particle resuspension;

- to highlight the limitations of the experimental characterisations of surface roughness;

- to identify and underline the refined statistical descriptions required.

For that purpose, the recent dynamic approach for particle resuspension is briefly recalled in Section 2. Then, existing experimental data on the characterisation of surface roughness are analysed in Section 3. To highlight the need for more detailed characterisations of surface roughness, numerical results obtained with the modelling approach are compared to recent experimental data in Section 4. These numerical results are then used to suggest a refined characterisation of surface roughness in Section 5.

\section{Present modelling approach}

\subsection{Existing models for particle resuspension}

Various modelling approaches have been developed in the literature. Since this article is focussed on assessing the role of surface roughness in particle resuspension and the limitations of the current measurements, only the key features of existing models are recalled here (the reader is referred to previous reviews for more details $[11,14,34]$ ).

Particle resuspension results from the intricate coupling between particle-fluid interactions and particle-surface interactions leading to a variety of possible mechanisms for parti-

cle resuspension (i.e. rolling, sliding and direct lift-off). The main difficulty in summarising existing modelling approaches is that they have been developed in different contexts and 


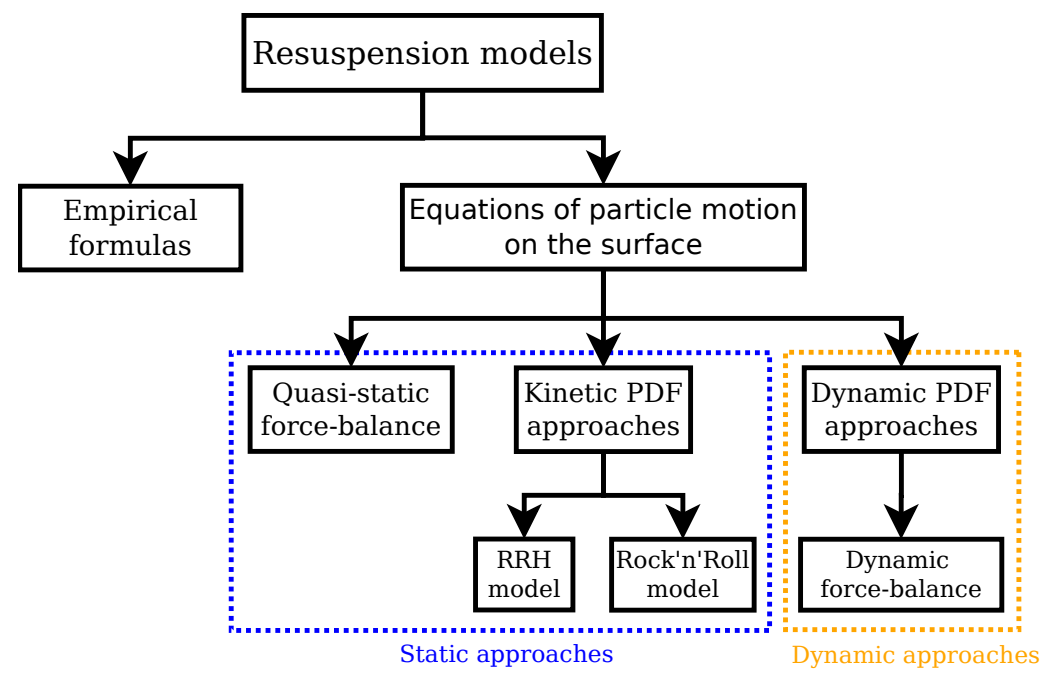

Figure 1: Sketch summarising the classification of modelling approaches for particle resuspension. Reprinted from [14]. Copyright 2014 with permission from Elsevier.

under various assumptions. As a result, it is not straightforward to suggest a classification that encompasses the whole range of possible approaches. Yet, a new classification has been suggested in a recent review [14]: as depicted in Fig. 1, a first distinction is made between empirical formulas which describe the overall effect of particle resuspension and modelling approaches where particle resuspension is estimated based on the equations of particle motion. A second distinction is made between various models: quasi-static force balance approaches (where resuspension is described as the rupture of equilibrium between particle-surface and particle-fluid forces as in [17, 18]), kinetic PDF approaches (where resuspension is described in a similar way to particles escaping from a potential well [26, 31]) and dynamic approaches (where the dynamics of particle rolling/sliding on a surface is accounted for $[12,16])$.

In the present paper, particle resuspension is addressed using the dynamic model developed previously $[12,16,15]$. This choice is mostly motivated by the fact that we focus on non-deformable colloidal particles whose motion is significantly affected by adhesion forces. Such colloidal particles are usually embedded within the viscous layer and resuspend mostly through rolling motion. 

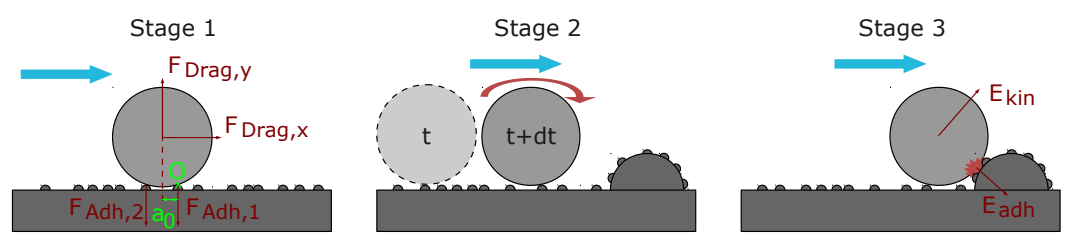

Figure 2: Sketch of the three-stage process for particle removal: particles are first set in motion (stage 1), then roll on the rough surface (stage 2) before being detached upon rocking on a large asperity (stage 3 ). Reprinted with permission from [16]. Copyright 2012 American Chemical Society.

\subsection{Description of the present modelling approach}

The dynamic model for particle removal has been described thoroughly in recent papers $[12,15,16]$. Therefore, only the main features of this stochastic model for particle removal are recalled in the following paragraphs.

The main idea behind this approach is to describe the removal of particles small enough to be well within the viscous sublayer. In that case, particle removal has been shown to occur mainly through rolling motion [14]. As depicted in Fig. 2, particle removal has been modelled considering a three-step process:

1. First, particles deposited on a surface are set into motion if there is a rupture of the balance between hydrodynamic forces (which tend to remove particles from surfaces) and adhesion forces (which act to prevent particles from moving).

Here, hydrodynamic forces acting on particles are considered to be given by drag forces only (gravity and lift forces are negligible for such small colloids). Meanwhile, adhesion forces between non-deformable particles are calculated using the DLVO theory which combines van der Waals and electrostatic double-layer interactions together (only van der Waals forces play a role here since particles are reentrained by an airflow). We also take into account surface roughness through a simplified representation where rough surfaces are modelled with a simple smooth substrate covered by hemispherical asperities. It should be noted here that, in the case where both particles and surfaces are rough, we combine both distributions together and assume that roughness is present on the substrate only. Then, we extract the moment of 
these adhesion forces using the calculated force and the tangential distance to the asperity in contact with the particle.

2. Second, particles roll on the surface, accelerating or decelerating depending on the hydrodynamic and adhesion forces/moments encountered along their trajectory.

The equation of particle translational motion are obtained from the equation of rotational motion and using a linear approximation to relate the particle angular velocity $\omega$ to the streamwise velocity: $U_{p, / /} \simeq R_{\text {part }} \omega$. This gives:

$$
I \frac{d U_{\text {part }, / /}}{d t} \simeq R_{\text {part }} M_{0}\left(F_{\text {drag }, / /}\right)-R_{\text {part }} M_{0}\left(F_{\text {adh }}\right)
$$

with $I=7 m_{\text {part }} R_{\text {part }}^{2} / 5$ the particle moment of inertia, $R_{\text {part }}$ the particle radius, $M_{0}\left(F_{d r a g, / /}\right)$ the moment of hydrodynamic drag forces and $M_{0}\left(F_{a d h}\right)$ the moment of adhesion forces. The equations of motion are solved every time step for every particle rolling on the surface.

3. Third, particles rolling on the surface can hit large-scale asperities present on the surface possibly leading to their detachment from the surface.

During these "rocking events", detachment occurs if the particle kinetic energy (considered to be oriented perpendicularly to the surface) is higher than the adhesion energy between the particle and the surface.

This three-step process aims at capturing the dynamics of rolling particles on a surface by making a distinction between particle detachment and particle resuspension. Here, detachment is the last step of the resuspension process, with the first step corresponding to the initiation of rolling motion due to the rupture of balance between hydrodynamic and adhesion moments. Accounting for the dynamics of rolling particles on the surface can prove key in the capture of particles in regions of the surface with high adhesion forces: this has been recently suggested for particles close to pore edges [8] and will be investigated in future studies. It also illustrates how hydrodynamic effects and physico-chemical effects (with very different spatial and temporal scales) can be coupled within a single modelling approach. 
Another important feature of the present modelling approach is that adhesion forces are calculated explicitly. For that purpose, rough surfaces are described by a smooth plane covered by hemispherical asperities having a range of radii and surface coverages. The distribution in the adhesion force with such rough surfaces is thus obtained as a result of Monte-Carlo simulations where rough surfaces are generated randomly using the roughness characteristics measured experimentally. This model for adhesion forces between non-deformable rough surfaces has been validated previously with measurements [16]: it has been shown to reproduce various forms of the adhesion force distribution, including Gaussian or log-normal distributions as well as more complex two-peaks distributions. Similarly, the whole model for particle removal has been shown to provide fairly good results compared to experimental data on the resuspension of small colloidal particles $[12,15,16]$.

\section{Measurement of surface roughness in the context of particle resuspension}

\subsection{Current approaches to measure roughness features}

The presence of geometric heterogeneities on surfaces has long been confirmed experimentally using either AFM (Atomic Force Microscope) or SEM (Scanning Electron Microscope) techniques to probe the surface. These measurement techniques provide $2 \mathrm{D}$ or $3 \mathrm{D}$ images of the surface revealing the complex topography of rough surfaces (more details can be found in [29]). In the context of particle adhesion and resuspension, several parameters characterising surface roughness are usually extracted, among which (see also Fig. 3):

- $R_{a}$, the mean roughness which corresponds to the arithmetic average of the roughness height (absolute) above a reference plane. For a 2D profile, it is defined as:

$$
R_{a}=\frac{1}{L} \int_{0}^{L}|Z(x)| d x
$$

- $R_{r m s}$, the root-mean-squared roughness which is the root mean square average of the roughness roughness profile coordinates. For a 2D profile, it is defined as:

$$
R_{r m s}=\sqrt{\frac{1}{L} \int_{0}^{L} Z(x)^{2} d x}
$$




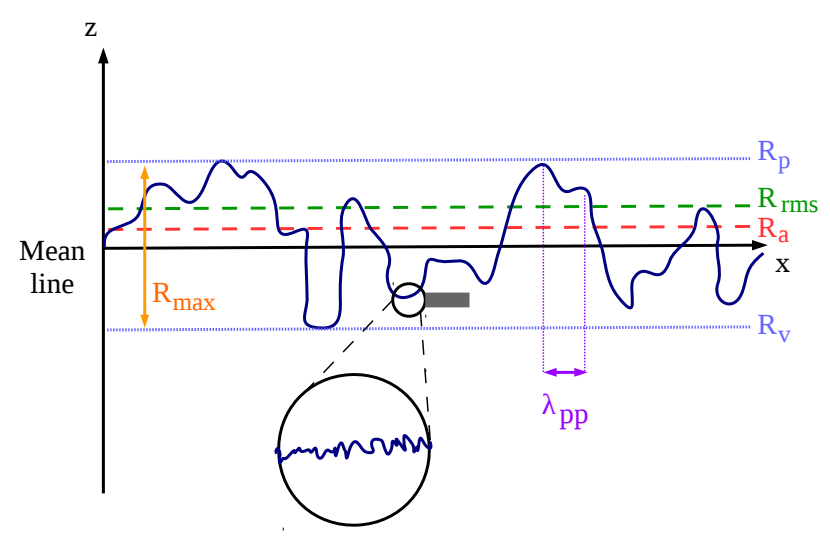

Figure 3: Sketch of a rough surface and the corresponding roughness parameters $\left(R_{a}\right.$ and $\left.R_{r m s}\right)$.

- $R_{v}$, the lowest valley of the surface profile. For a 2D profile, it is defined as:

$$
R_{v}=\min Z(x)
$$

- $R_{p}$, the highest peak of the surface profile. For a 2D profile, it is defined as:

$$
R_{p}=\max Z(x)
$$

- $R_{\max }$, the maximum height of the profile. For a 2D profile, it is defined as:

$$
R_{\max }=R_{p}+R_{v}
$$

- $R_{z}$ the average distance between the highest peak and the lowest valley over a set of $n_{\text {set }}$ samples each with a sampling length $l_{\text {set }}$. For a 2D profile, it is defined as:

$$
R_{z}=\frac{1}{n_{s e t}} \sum_{i=1}^{n_{s e t}}\left(R_{p, i}+R_{v, i}\right)
$$

- $\lambda_{p p}$, the peak-to-peak distance which is the mean distance between two consecutive asperities (related to the surface coverage of asperities on the surface).

These parameters are extensively used since they provide statistical information on roughness characteristics that are easy to measure. However, these parameters only provide a limited amount of information. The difficulty that arises when trying to fully characterise 
a rough surface is that the surface features present on the surface exhibit a very large range of sizes and surface coverage (except in some cases where surfaces are designed on purpose to display specific roughness features). In the following, we list the limitations of these current measurements:

1. Lack of spatial information:

Most of the parameters listed previously do characterise a rough surface in terms of the height of the asperities present on the surface and only $\lambda_{p p}$ does provide information on the spatial distribution of these asperities. This information is key in describing surface roughness since the surface covered by asperities (or their density) has a direct impact on the number of contacts that can exist between two adhering rough surfaces. It is worth mentioning that, in the case where the asperities on the surface have a spherical shape (i.e. the asperity height is equal to the curvature radius), the surface coverage is actually related to the distribution (which is a measure of the frequency of occurrence). Yet, this case is not always likely due to the chaotic nature of surface roughness.

2. Homogeneity of surface roughness:

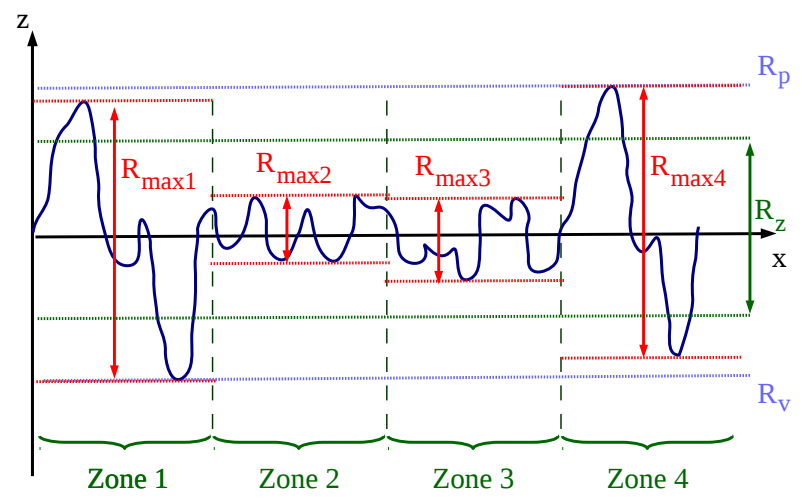

Figure 4: Sketch of a rough surface and the corresponding roughness parameters $\left(R_{z}\right)$.

These parameters implicitly require that the sampling area is spatially homogeneous (i.e. that changing the area of the sample zone does not modify the statistics). This is illustrated in Fig. 4 which displays a heterogeneously rough substrate: it can be 
seen that the regions labelled 2 and 3 (respectively 1 and 4) display similar surface features since $R_{\max , 2} \simeq R_{\max , 3}\left(\right.$ resp. $\left.R_{\max , 1} \simeq R_{\max , 4}\right)$. Yet, the size of the largest surface features in regions 2 and 1 are different. As a result, the value of $R_{z}$ depends on the size of the region sampled. In the example depicted in Fig. 4, it can be seen that the information on $R_{\max , 2} \simeq R_{\max , 3}$ will be unavailable if these statistical parameters are extracted from the whole field of view.

3. Lack of information on the distribution in roughness features:

The chaotic nature of surface roughness often leads to the presence of surface features with a large range of sizes and surface coverage. Yet, the level of information is reduced to the knowledge of a few given statistical parameters related to the mean size of roughness features. There is thus no information on the exact distribution in the size of surface features (except $R_{z}$ and $R_{\max }$ which give an idea of the largest surface features).

This lack of detailed information on the exact size distribution and spatial repartition of surface features limits the accuracy of models for adhesion forces between rough surfaces. The knowledge of the average size of roughness features can only provide satisfactory predictions of the average adhesion forces but not of the overall distribution.

\subsection{Selected case of colloid resuspension from rough substrates}

In the following, we illustrate how this lack of detailed information on the exact size distribution and spatial repartition of surface features affects numerical predictions by selecting a specific experimental study dealing with colloid resuspension.

For that purpose, we have chosen to retain the recent measurements of small colloidal particles resuspended from rough substrates in a turbulent channel airflow at various friction velocities $u_{\tau}$ obtained by Barth et al. [2]. This choice is motivated by the fact that the motion of small colloidal particles is significantly affected by adhesion forces and thus by surface roughness.

In that experiment, the number of particles remaining on a wall segment in a horizontal square duct has been recorded after a one-minute exposure to a turbulent flow with a 


\begin{tabular}{|c|c|c|c|}
\hline Sample & $R_{a}(\mu \mathrm{m})$ & $R_{z}(\mu \mathrm{m})$ & $R_{\max }(\mu \mathrm{m})$ \\
\hline Smooth glass plate & 0.025 & 0.220 & 0.477 \\
\hline
\end{tabular}

Table 1: Surface roughness specifications of different rough substrates used in [2].

\begin{tabular}{|c|c|c|c|c||c|c|}
\hline Case & $\begin{array}{c}d_{p} \\
(\mu \mathrm{m})\end{array}$ & $\sigma_{\text {geom }}$ & circularity & $\begin{array}{c}\rho_{p} \\
\left(\mathrm{~kg} / \mathrm{m}^{3}\right)\end{array}$ & $\begin{array}{c}\nu_{f} \\
\left(\mathrm{~m}^{2} / \mathrm{s}\right)\end{array}$ & $\begin{array}{c}\rho_{f} \\
\left(\mathrm{~kg} / \mathrm{m}^{3}\right)\end{array}$ \\
\hline GBM 20 & 25 & 1.15 & 1 & & & \\
GBM 30 & 35 & 1.10 & 1 & 4100 & $1.56 \times 10^{-5}$ & 1.178 \\
GBM 40 & 44 & 1.07 & 1 & & & \\
\hline
\end{tabular}

Table 2: Particles and fluid (air) properties

gradual increase of the fluid velocity [2]. Two types of particles were used in the experiment:

- spherical barium-titanate glass particles (GBM) with a geometric diameter ranging from $25 \mu \mathrm{m}$ up to $44 \mu \mathrm{m}$ with small dispersion (geometric standard deviation smaller than 1.15).

- polypropylene particles with a geometric diameter of $11.8 \mu \mathrm{m}$ with some dispersion (geometric standard deviation equal to 1.9).

To evaluate the effect of surface roughness on particle removal, the authors have also used two types of substrates: either a smooth glass plate or steel plates with various roughness characteristics.

To avoid introducing additional complexity, we focus here on the case of GBM particles resuspended from glass substrates. This choice is motivated by the fact that these particles are monodispersed and spherical (circularity equal to 1) and that the glass substrates are relatively smooth (with possibly less complex distributions in the size of roughness features present on the surface). Tables 1 and 2 summarise the roughness characteristics of the glass substrates measured using AFM, the properties of the GBM particles and of the fluid (air). 


\section{Analysis of numerical results for particle resuspension}

In the following, we compare numerical results obtained with the stochastic approach described in Section 2 to these recent experimental data on particle resuspension. Numerical results are used to illustrate how the limitations in the current characterisations of surface roughness affect the accuracy of modelling approaches and their predictive abilities.

\subsection{Numerical parametrisation}

Numerical procedure. Following the experimental procedure, numerical simulations are performed by exposing particles to a turbulent flow with a given fluid velocity for $60 \mathrm{~s}$ and calculating the number of particles remaining on the surface at the end of the simulation. All simulations have been performed using a time step of $10^{-4} \mathrm{~s}$ and a Hamaker constant for the glass-glass system $A_{\text {Ham }}=10 \times 10^{-20} \mathrm{~J}$. The particle and fluid properties have been set to the values indicated in Table 2 .

Description of rough surfaces. As mentioned previously in Section 3.1, experimental results provide only limited information on surface roughness. There are two main difficulties that arise when trying to generate numerically rough surfaces with the same characteristics:

- First, the choice of a rough surface is not unique. Indeed, the model for adhesion forces is based on a simplified description of rough surfaces where hemispherical asperities cover a smooth plate. In such cases, the typical roughness characteristics $\left(R_{a}, R_{r m s}\right)$ depends on both the size of the hemispherical asperities and their spatial distribution (the exact derivation of the formula for $R_{a}$ is given in the Appendix). As a result, rough surfaces can be generated numerically with very different values for the asperity size and surface coverage but with the same values for the overall roughness characteristics $\left(R_{a}\right)$. This confirms that the knowledge of $R_{a}, R_{z}$ and $R_{r m s}$ does not fully characterise a rough surface.

- Second, in dynamic resuspension models such as the one used here, a distinction is made between small-scale asperities and large-scale asperities. The notion of small 


\begin{tabular}{|c||c|c|c|c||c|c|}
\hline Case & $\begin{array}{c}R_{\text {asp }}^{\text {small }} \\
(\mathrm{nm})\end{array}$ & $\begin{array}{c}S_{\text {cov }}^{\text {small }} \\
(\%)\end{array}$ & $\begin{array}{c}R_{\text {asp }}^{\text {large }} \\
(\mu \mathrm{m})\end{array}$ & $\begin{array}{c}S_{\text {cov }}^{\text {large }} \\
(\%)\end{array}$ & $\begin{array}{c}R_{a} \\
(\mathrm{~nm})\end{array}$ & $\begin{array}{c}R_{\text {max }} \\
(\mu \mathrm{m})\end{array}$ \\
\hline $\mathrm{R} 1$ & 9 & 3.14 & 0.5 & 3.97 & 25.3 & 0.5 \\
$\mathrm{R} 2$ & 9 & 3.14 & 0.22 & 9.4 & 24.6 & 0.22 \\
$\mathrm{R} 3$ & 8 & 2.48 & 0.5 & 3.97 & 25.3 & 0.5 \\
$\mathrm{R} 4$ & 8 & 2.48 & 0.22 & 9.4 & 24.5 & 0.22 \\
$\mathrm{R} 5$ & 25 & 1.91 & 0.5 & 3.97 & 25.3 & 0.5 \\
\hline
\end{tabular}

Table 3: Set of values for the roughness properties in each case studied numerically

and large asperities has to regarded with respect to the diameter of deposited particles and is thus relative to a particle size. This distinction between small- and large-scale asperities has proved useful since their role is very different [15, 16]: small-scale asperities directly impact the adhesion forces while large-scale asperities impact the dynamics of rolling particles. Yet, the knowledge of $R_{a}$ or $R_{r m s}$ is insufficient to provide information on the asperity size and on the surface coverage for both small and large-scale asperities. In the present case, the size of the large-scale surface features has been chosen close to either $R_{\max }$ or $R_{z}$ (since both values characterise the largest features on the scanned surface). Meanwhile, the size of small-scale features is not available in the present measurements and has been chosen either equal to $25 \mathrm{~nm}$ (a value close to the mean roughness $R_{a}$ ) or close to $9 \mathrm{~nm}$ (since it provides better results). The surface covered by these surface features has been chosen to respect the value $R_{a}$ measured experimentally. Table 3 summarises the various set of data for small- and large-scale asperities used in the present simulations.

\subsection{Numerical results}

In the following, we compare numerical results to experimental data in an attempt to further highlight the role of surface roughness and the need for refined characterisations of surface roughness. 




(a) Case R1

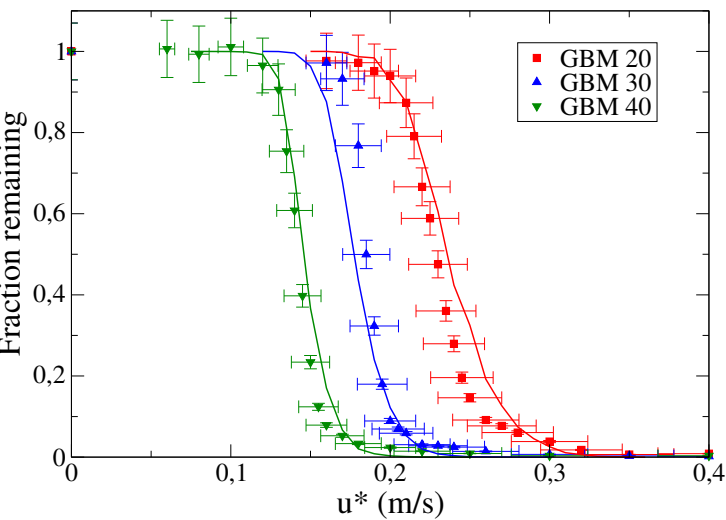

(b) Case R2

Figure 5: Fraction of GBM particles remaining on a smooth glass substrate against the friction velocity: experimental data (symbols and error bars), numerical results (lines) obtained for cases R1 (left) and R2 (right).

Effect of large-scale asperities. Fig. 5a displays the numerical results obtained for case R1. It can be seen that these results are in good agreement with experimental data regardless of the GBM particle size, except at very large values of the friction velocity where numerical results tend to overestimate the resuspension rate (the origin of this overestimation will be discussed later in Section 5.1). As depicted in Fig. 5b for case R2, changing the size (and surface coverage) of large-scale asperities to values equal to $R_{z}$ instead of $R_{\max }$ also provides satisfactory results regardless of the GBM particle size. These results confirm that the characteristics of large-scale asperities do not have a significant effect on particle resuspension. This is due to the fact that large-scale asperities impact the frequency of detachment due to rocking events in the present modelling approach but do not greatly affect the adhesion forces.

Effect of small-scale asperities. Fig. 6 displays numerical results obtained for cases R3 and $\mathrm{R} 4$, which are identical to cases $\mathrm{R} 1$ and $\mathrm{R} 2$ except that the size and surface coverage of small-scale asperities has been slightly changed (here from $9 \mathrm{~nm}$ to $8 \mathrm{~nm}$ ) while respecting the mean roughness $R_{a}$. It can be seen that the numerical results are also in good agreement with experimental data regardless of the GBM particle size, especially for case 


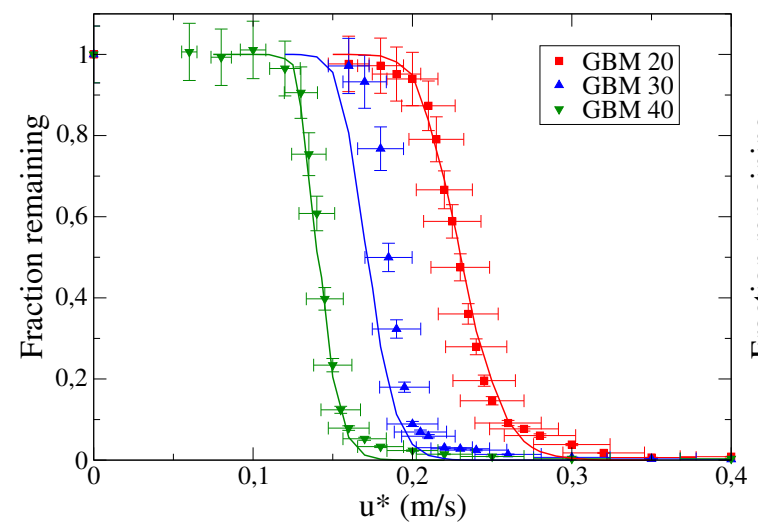

(a) Case R3

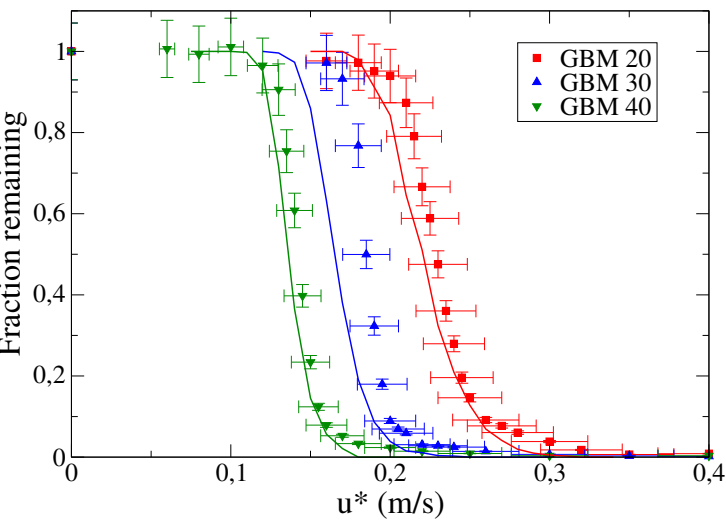

(b) Case R4

Figure 6: Fraction of GBM particles remaining on a smooth glass substrate against the friction velocity: experimental data (symbols and error bars), numerical results (lines) obtained for cases R3 (left) and R4 (right).

R3. Comparing these results to the cases R1 and R2, it appears that a slight change of the characteristics of small-scale asperities can already have a noticeable impact on the resuspension rate. This is due to the fact that small-scale asperities govern particle-surface adhesion forces [16] and can thus have a profound impact on particle resuspension.

This is further illustrated in Fig. 7 (case R5) where the size of small-scale asperities has been increased to $25 \mathrm{~nm}$ : in that case, even though the mean roughness $R_{a}$ is the same as the one measured experimentally, it can be seen that numerical results severely underestimate the resuspension rate due to much higher adhesion forces between the particle and the rough substrate. This can be understood since adhesion forces are directly proportional to the size of small-scale asperities. The broad distribution of adhesion forces that results from the interaction with a rough substrate is thus responsible for the fact that resuspension occurs over a wide range of friction velocities. Assuming that particles have all the same size, particles encountering smaller adhesion forces will indeed be removed at smaller fluid velocities.

Main results. The main conclusions that can be drawn from these results are two-fold:

- First, numerical results can provide correct predictions of the overall shape of the 


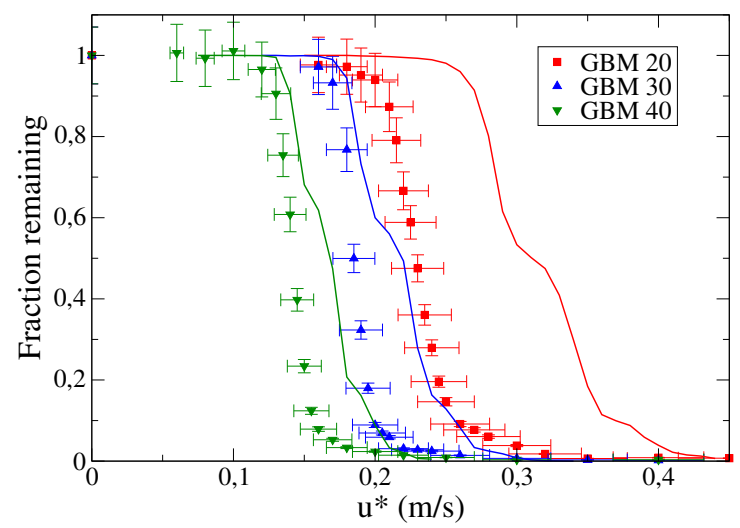

Figure 7: Fraction of GBM particles remaining on a smooth glass substrate against the friction velocity: experimental data (symbols and error bars), numerical results (lines) obtained for case R5.

resuspension rate and especially of $u_{50}^{*}$ (the friction velocity at which $50 \%$ of the particles are resuspended) but tend to overestimate the resuspension rate at high friction velocities.

- Second, there is actually a range of values for the size and surface coverage of smallscale asperities that can explain the observed behaviour of particle resuspension. Therefore, the characterisation of rough surfaces using only a limited amount of information (here $R_{a}, R_{z}$ and $R_{\max }$ ) is insufficient and more detailed information on the distribution of asperity sizes and densities should be extracted from surface profiles.

\section{Discussion: towards refined descriptions of surface roughness}

As mentioned in the Introduction, understanding and predicting particle resuspension over the whole range of friction velocities encountered is sometimes a key issue (for instance to avoid resuspension of radioactive particles). In such cases, predicting the average properties of particle resuspension (such as $u_{50}^{*}$ ) is not satisfactory enough since particle resuspension can also occur at much higher/lower velocities. Drawing on such needs to predict the overall resuspension rate, we attempt here to provide clues to answer the following question: "which additional information on surface roughness is needed to provide 
more accurate simulations of particle adhesion and resuspension?"

\subsection{Insights into additional information needed}

If we focus on the high-velocity tails of the resuspension rates, it appears that experimental results show a non-zero fraction of particles remaining on the surface whereas numerical results predict a fraction of particles remaining on the surface equal to zero in those cases (see Figs. 5 and 6). This discrepancy can be attributed to an underestimation of the highest values of the moments of adhesion force. To investigate this effect, we have performed additional simulations by modifying the tail of the adhesion force distribution in case R2: the $2 \%$ particles undergoing the highest moment of adhesion forces in the simulation have been modified to encounter a moment of adhesion forces multiplied by a factor taken randomly between 1 and 3. Numerical results obtained for GBM 40 particles are displayed in Fig. 8, where several features can be seen:

- the resuspension rate is unchanged compared to case R2 at small friction velocities since only the tail of the distribution of adhesion forces has been modified;

- a non-zero fraction of particles remains on the surface for friction velocities between $0.18 \mathrm{~m} / \mathrm{s}$ and $0.28 \mathrm{~m} / \mathrm{s}$ since the small fraction of particles encountering higher adhesion forces and moments are much harder to set in motion by the fluid;

- no particles remain on the surface for friction velocities higher than $0.35 \mathrm{~m} / \mathrm{s}$ since the moment of hydrodynamic forces always overcomes the moment of adhesion forces obtained here.

These results further highlight the fact that correct predictions of the resuspension rate over the whole range of friction velocities can only be obtained with a proper calculation (or measurement) of the whole distribution in adhesion forces and moments. In particular, the tail of the distribution in the resuspension rate is related to the tail of the distribution in the moment of adhesion forces since particles encountering higher adhesion forces/moments are harder to remove. From a physical point of view, the increased adhesion forces/moments encountered by a fraction of particles in the present case can be attributed to two sources: 


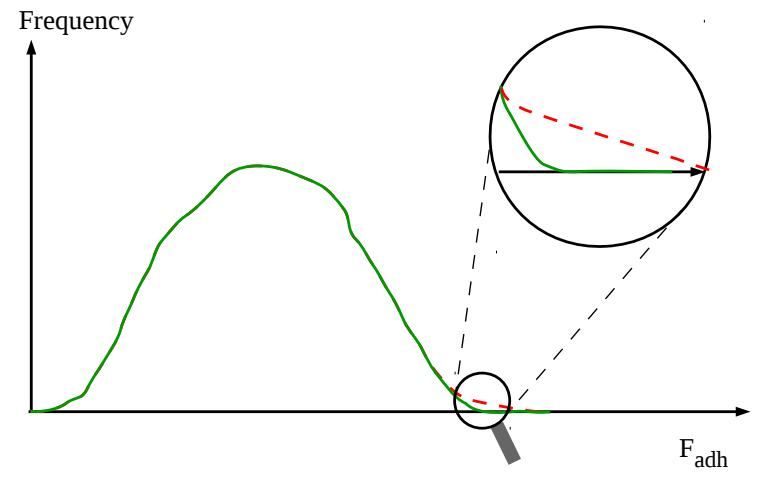

(a) Sketch showing a modified tail of $F_{a d h}$

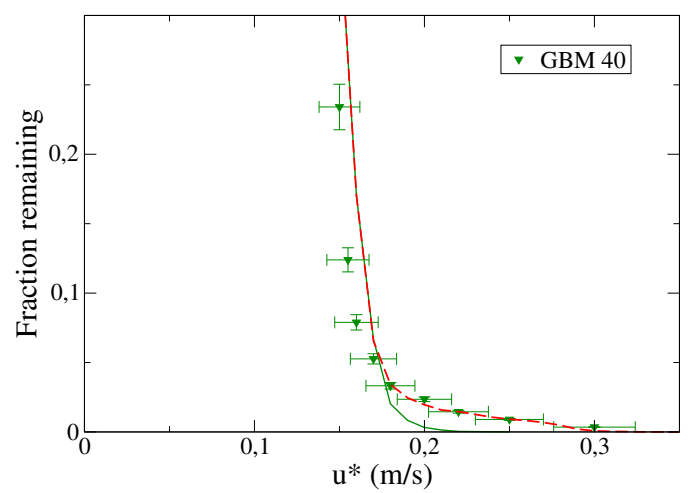

(b) Effect on the resuspension rate

Figure 8: Fraction of GBM 40 particles remaining on a smooth glass substrate against the friction velocity: experimental data (symbols and error bars), numerical results (lines) obtained for case R2 alone (green line) and with a modified tail of the distribution in moment of adhesion forces (red dashed line).

1. first, a higher adhesion force/moment is experienced by particles in contact with larger asperities. Here, the adhesion force is given by van der Waals forces only and thus directly proportional to the asperity radius. Therefore, the small fraction of particles undergoing three-time higher adhesion forces/moments can be related to the presence of asperities three times bigger (around $25 \mathrm{~nm}$, i.e. close to the value of $R_{a}$ measured). This possibility further confirms that measuring the distribution in the size of surface features is key in understanding and modelling particle resuspension.

2. second, a higher adhesion force/moment can also be obtained if the surface coverage of asperities is locally higher. In the present modelling approach, the number of asperities in contact with the particles is indeed proportional to the surface coverage. Therefore, if a local patch contains asperities with a surface coverage three times higher than the average, particles interacting with such patches will experience an adhesion force/moment roughly three times higher. This possibility underlines the role that can be played by spatial heterogeneities in surface roughness features. As mentioned in Section 3, statistical measurements of surface roughness (either through simple statistical values or more refined distributions) do make sense only if they are spatially homogeneous. Therefore, upon extracting statistical roughness character- 
istics from surface profiles, great care must be taken to verify that these statistics characterise spatially homogeneous regions of the surface (i.e. that changing the area of the sample zone does not modify the statistics). In the case of heterogeneous substrates, several characterisations will be needed for each type of patches encountered on the surface.

\subsection{A refined characterisation of surface roughness for particle resuspension}

Numerical results have shown that, since particles are set into motion when the balance between hydrodynamic and adhesion forces is ruptured, resuspension at low/high velocities is governed by the tail in the distribution of adhesion forces and thus by the tail in the distribution of surface roughness. Drawing on these results and on recent experimental studies, we provide a first tentative answer to the need for refined characterisations of surface roughness in the context of particle resuspension.

Curvature radius. Recent findings have revealed that the key parameter affecting adhesion forces is not the characteristic height of surface features but their curvature radius $[16,20,24]$. This is due to the fact that adhesion forces are often governed by van der Waals forces. Due to these short-ranged van der Waals forces, the interaction between two bodies in contact is mostly governed by the two volumes directly facing each other (see the blue regions in Fig. 9). Thus, adhesion forces are sensitive to the curvature radius

of the roughness features present on the surfaces in contact and not necessarily to the characteristic roughness height.

Recent measurements of surface roughness in the context of particle adhesion have shown that it is possible to extract the curvature radius of asperities present on the surface from the surface profile (see $[24,20]$ ). For instance, as depicted in Fig. 10, Prokopovich and Perni have provided the whole distribution of asperity heights and curvature radii.

Distributions of roughness features. Dynamic approaches have shown that the role of surface roughness is two-fold $[14,16]$ : small-scale surface features directly impact the adhesion forces while large-scale surface features play a role in the dynamics of rolling particles. It is 


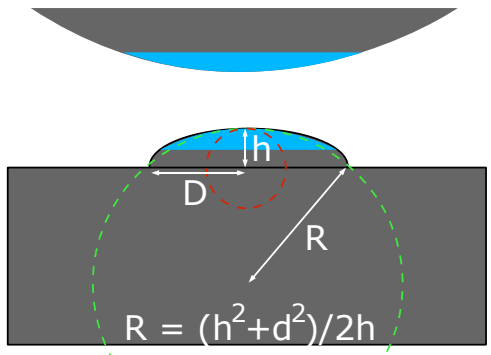

(a) Broad ellipsoidal asperities

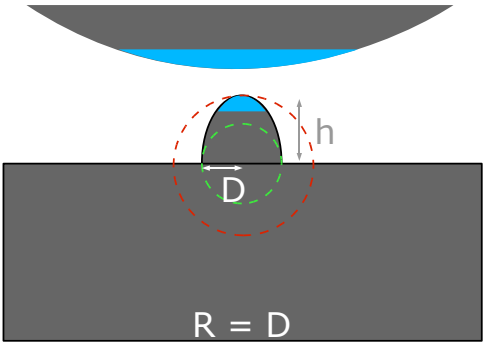

(b) Slender ellipsoidal asperities

Figure 9: Illustration of the role of the curvature radius on adhesion forces: the shaded blue regions facing each other . Reprinted from [16]. Copyright 2012 with permission from American Chemical Society.

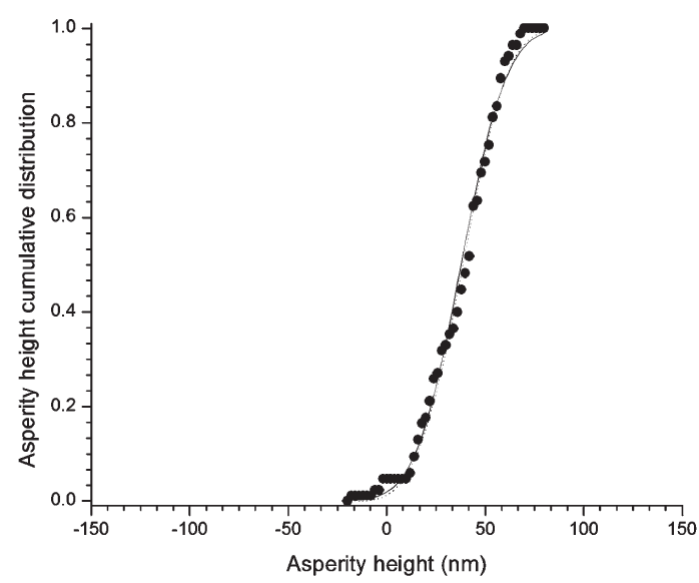

(a) Asperity height

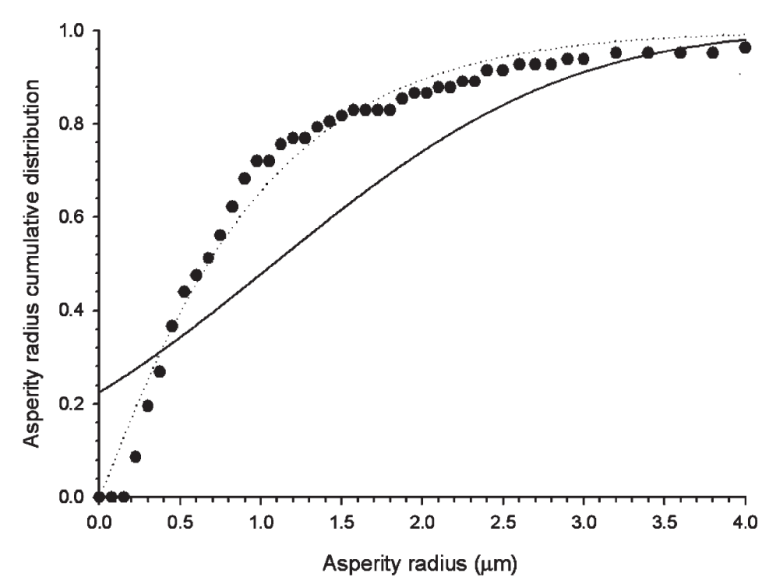

(b) Asperity curvature radius

Figure 10: Cumulative distribution of asperity height (left) and curvature radius (right) of a PVC substrate: experimental data (black dots) and fits (dotted line). Taken from [24]. Copyright 2010 with permission from the American Chemical Society. 
important to note that the notion of small and large scales is relative to a particle diameter and, therefore, can vary with respect to particle sizes. As a result, the refined characterisation should include details on both the small-scale and large-scale surface features. Thus, the refined characterisation cannot be limited to the usual knowledge of average parameters ( $R_{a}, R_{r m s}$, etc.) completed with the smallest and largest roughness features.

Numerical results have also shown that the refined characterisation should include information on both the size of surface features and their spatial correlation (surface coverage). The size (curvature radius) of surface features will indeed directly affect the adhesion forces while the surface coverage can change the number of contact between both surfaces. This is in line with recent experimental and numerical studies on the effect of surface roughness on adhesion forces $[1,5,7,9,20,25,32]$.

Drawing on these remarks, it appears that the key information needed to describe surface roughness in the context of particle resuspension is the distribution of both asperity curvature radii and the corresponding surface coverage of asperities on the surface for spatially homogeneous regions of the surface. This is depicted in Fig. 11, which also displays the specific role of small-scale and large-scale surface features in resuspension through rolling motion. An important feature to be noted here is that these distributions should be as complete as possible (for instance using logarithmic scales) since the tail of these distributions can have a significant impact on adhesion forces and thus resuspension.

\section{Conclusion}

The present paper proposes new insights into the issue of the resuspension of small colloidal particles from rough surfaces through rolling motion. A brief analysis of the existing measurements of surface roughness has brought out the limitations in the standard characterisation of roughness in terms of statistical values (such as $R_{a}, R_{\max }, R_{z}, \lambda_{p p}$, etc.). Numerical results obtained with a dynamic model for particle resuspension due to rolling motion have been compared to recent experimental data for short-term resuspension. These numerical results have further highlighted the role played by surface roughness [16], where small-scale and large-scale roughness have a different role: 


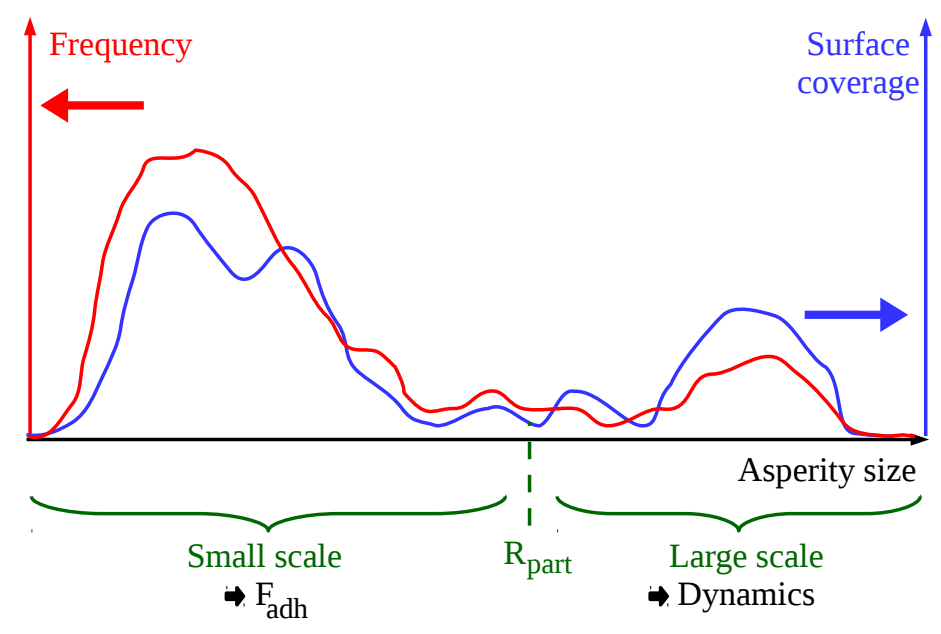

Figure 11: Sketch showing a possible distribution in asperity curvature radius and surface coverage needed to obtained a refined characterisation of surface roughness in the context of particle resuspension.

1. large-scale asperities impact the detachment step (in agreement with the scenario for particle resuspension retained here) and have thus a limited impact on the resuspension rate;

2. small-scale asperities significantly affect the adhesion force and moment of force between the particles and the surface, thus strongly influencing the rate of particle resuspension.

Besides, numerical results have shown that a range of values for the size and surface coverage of small-scale asperities can explain the observed behaviour of particle resuspension, confirming the limitations of the present characterisation of rough surfaces and the need for refined measurements. In particular, additional simulations have shown that resuspension of particles at high friction velocities is related to the tail of the distribution of adhesion forces and thus to the tail of the distribution of roughness features.

Drawing on these results, a refined characterisation of surface roughness has been suggested to better predict the effect of surface roughness on adhesion forces and particle resuspension: 
- First, to go beyond the present limitations, the authors believe that measurements of the curvature radius of surface features should be extracted from surface profiles (and not only of the height above a reference plate) since it has a significant impact on adhesion forces.

- Second, information on the distribution of curvature radii and their spatial correlations (surface coverage) should be provided.

- Third, such refined characterisations of surface roughness should only be extracted in spatially homogeneous regions of the surface (i.e. changing the sampling area does not change the statistics). Therefore, the presence of local patches (due to some spatial heterogeneity of the surface) should be characterised separately.

The authors are of the opinion that refined predictions of particle resuspensions will be possible thanks to future experimental data that provide such detailed characterisation of the surface topology. Future experimental studies should also provide information on the adhesion force distribution as well as the resuspension rate measured using this given particle-substrate system for completeness. With such refined measurements, it will also be possible to study specifically the case of long-term resuspension, which is highly affected by the tail of the distribution in adhesion forces (where rare extreme events take place on longer times). We believe that such fine calculations of the adhesion force distribution and of the corresponding resuspension rate will provide very useful information for more macroscopic models in particle resuspension (where adhesion forces or resuspension rates are source terms).

\section{Acknowledgement}

The authors acknowledge the EU COST Action MP1305, supported by COST (European Cooperation in Science and Technology). 


\section{Appendix}

Calculation of the roughness parameters in the simulation

In the present simulations, a rough surface is described using a smooth plate covered by hemispherical asperities. In the following, we thus consider hemispherical asperities (radius $\left.R_{a s p}\right)$ with a given surface coverage $\left(S_{\text {cov }}\right)$. In that case, the reference plane is located at the mean height above the smooth plate. To evaluate this mean height, we first consider the case of a single asperity present on the surface. In that case, the mean height is given by the following integration:

$$
R_{0,1 a s p}=\frac{1}{\pi R_{a s p}^{2}} \int_{0}^{R_{a s p}} 2 \pi z \sqrt{R_{a s p}^{2}-z^{2}} d z
$$

which integrates as

$$
R_{0,1 a s p}=\frac{2}{3} R_{a s p}
$$

As a result, when considering an entire rough surface with hemispherical asperities covering a certain amount of the surface, the reference plate is at a height $R_{0}$ given by

$$
R_{0}=\frac{2}{3} R_{a s p} \times S_{c o v}
$$

The mean roughness $R_{a}$ is then obtained by integrating the absolute roughness height above the reference plate. For a single asperity, this amounts to:

$$
R_{a, 1 a s p}=\frac{1}{\pi R_{a s p}^{2}} \int_{0}^{R_{a s p}} 2 \pi\left|z-R_{0}\right| \sqrt{R_{a s p}^{2}-z^{2}} d z
$$

Depending on the relative size between the asperity height and the height of the reference plate, this integrates as:

$$
\begin{aligned}
& \text { if } R_{a, 1 a s p}>R_{a s p} \text { then } \\
& \qquad \begin{aligned}
R_{a, 1 a s p}= & \frac{2}{3} R_{a s p}\left[2\left(1-\frac{R_{0}^{2}}{R_{a s p}^{2}}\right)^{3 / 2}-1\right] \\
& +2 R_{0}\left[\operatorname{atan}\left(\frac{R_{0}}{\sqrt{R_{a s p}^{2}-R_{0}^{2}}}\right)+\frac{R_{0}^{2}}{R_{a s p}^{2}} \sqrt{\frac{R_{a s p}^{2}}{R_{0}^{2}}-1}-\frac{\pi}{4}\right]
\end{aligned}
\end{aligned}
$$

if $R_{a, 1 a s p}<R_{a s p}$ then

$$
R_{a, 1 a s p}=R_{0} \frac{\pi}{2}-\frac{2}{3} R_{a s p}
$$


In the general case (entire rough surface with hemispherical asperities), the average roughness is:

$$
R_{a}=R_{a, 1 a s p} \times S_{c o v}+\left(1-S_{c o v}\right) \times R_{0}
$$

\section{References}

[1] Audry, M.-C., Ramos, S., \& Charlaix, E. (2009). Adhesion between highly rough alumina surfaces: an atomic force microscopy study. Journal of Colloid and Interface Science, 331, 371-378.

[2] Barth, T., Preuß, J., Müller, G., \& Hampel, U. (2014). Single particle resuspension experiments in turbulent channel flows. Journal of Aerosol Science, 71, 40-51.

[3] Boor, B. E., Siegel, J. A., \& Novoselac, A. (2013). Monolayer and multilayer particle deposits on hard surfaces: literature review and implications for particle resuspension in the indoor environment. Aerosol Science and Technology, 355, 417-422.

[4] Braaten, D. A. (1994). Wind tunnel experiments of large particle reentrainmentdeposition and development of large particle scaling parameters. Aerosol Science and Technology, 21, 157-169.

[5] Brant, J. A., \& Childress, A. E. (2004). Colloidal adhesion to hydrophilic membrane surfaces. Journal of Membrane Science, 241, 235-248.

[6] Coleman, S. E., \& Nikora, V. I. (2008). A unifying framework for particle entrainment. Water Resources Research, 44, W04415.

[7] Cooper, K., Gupta, A., \& Beaudoin, S. (2000). Substrate morphology and particle adhesion in reacting systems. Journal of Colloid and Interface Science, 228, 213-219.

[8] Duru, P., \& Hallez, Y. (2015). A three-step scenario involved in particle capture on a pore edge. Langmuir, 31, 8310-8317. 
[9] George, M., \& Goddard, D. T. (2006). The characterisation of rough particle contacts by atomic force microscopy. Journal of Colloid and Interface Science, 299, 665-672.

[10] Götzinger, M., \& Peukert, W. (2004). Particle adhesion force distributions on rough surfaces. Langmuir, 20, 5298-5303.

[11] Gradoń, L. (2009). Resuspension of particles from surfaces: technological, environmental and pharmaceutical aspects. Advanced Powder Technology, 20, 17-28.

[12] Guingo, M., \& Minier, J.-P. (2008). A new model for the simulation of particle resuspension by turbulent flows based on a stochastic description of wall roughness and adhesion forces. Journal of Aerosol Science, 39, 957-973.

[13] Hall, D., \& Reed, J. (1989). The time dependence of the resuspension of particles. Journal of Aerosol Science, 20, 839-842.

[14] Henry, C., \& Minier, J.-P. (2014). Progress in particle resuspension from rough surfaces by turbulent flows. Progress in Energy and Combustion Science, 45, 1-53.

[15] Henry, C., \& Minier, J.-P. (2014). A stochastic approach for the simulation of particle resuspension from rough substrates: model and numerical implementation. Journal of Aerosol Science, 77, 168-192.

[16] Henry, C., Minier, J.-P., \& Lefèvre, G. (2012). Numerical study on the adhesion and reentrainment of nondeformable particles on surfaces: the role of surface roughness and electrostatic forces. Langmuir, 28, 438-452.

[17] Ibrahim, A. H., Dunn, P. F., \& Brach, R. M. (2003). Microparticle detachment from surfaces exposed to turbulent air flow: controlled experiments and modeling. Journal of Aerosol Science, 34, 765-782.

[18] Ibrahim, A. H., Dunn, P. F., \& Qazi, M. F. (2008). Experiments and validation of a model for microparticle detachment from a surface by turbulent air flow. Journal of Aerosol Science, 39, 645-656. 
[19] Jiang, Y., Matsusaka, S., Masuda, H., \& Qian, Y. (2008). Characterizing the effect of substrate surface roughness on particle-wall interaction with the airflow method. Powder Technology, 186, 199-205.

[20] Katainen, J., Paajanen, M., Ahtola, E., Pore, V., \& Lahtinen, J. (2006). Adhesion as an interplay between particle size and surface roughness. Journal of Colloid and Interface Science, 304, 524-529.

[21] Kim, Y., Gidwani, A., Wyslouzil, B. E., \& Sohn, C. W. (2010). Source term models for fine particle resuspension from indoor surfaces. Building and Environment, 45, $1854-1865$.

[22] Kobayakawa, M., Kiriyama, S., Yasuda, M., \& Matsusaka, S. (2015). Microscopic analysis of particle detachment from an obliquely oscillating plate. Chemical Engineering Science, 123, 388-394.

[23] Kubota, Y., \& Higushi, H. (2013). Aerodynamic particle resuspension due to human foot and model foot motions. Aerosol Science and Technology, 47, 208-217.

[24] Prokopovich, P., \& Perni, S. (2010). Multiasperty contact adhesion model for universal asperity height and radius of curvature distributions. Langmuir, 26, 17028-17036.

[25] Rabinovich, Y. I., Adler, J. J., Ata, A., Singh, R. K., \& Moudgil, B. M. (2000). Adhesion between nanoscale rough surfaces: II. Measurement and comparison with theory. Journal of Colloid and Interface Science, 232, 17-24.

[26] Reeks, M. W., \& Hall, D. (2001). Kinetic models for particle resuspension in turbulent flows: theory and measurement. Journal of Aerosol Science, 32, 1-31.

[27] Stempniewicz, M. M., \& Komen, E. M. J. (2010). Comparison of several resuspension models against measured data. Nuclear Engineering and Design, 240, 1657-1670.

[28] Wagenpfeil, F., Paretzke, H., Peres, J., \& Tschiersch, J. (1999). Resuspension of coarse particles in the region of chernobyl. Atmospheric Environment, 33, 3313-3323. 
[29] Whitehouse, D. (2012). Surfaces and their Measurement. Butterworth Heinemann.

[30] Yang, S., Zhang, H., \& Hsu, S. M. (2007). Correction of random surface roughness on colloidal probes in measuring adhesion. Langmuir, 23, 1195-1202.

[31] Zhang, F., Reeks, M., \& Kissane, M. (2013). Particle resuspension in turbulent boundary layers and the influence of non-Gaussian removal forces. Journal of Aerosol Science, $58,103-128$.

[32] Zhou, S., Götzinger, M., \& Peukert, W. (2003). The influence of particle charge and roughness on particle-substrate adhesion. Powder Technology, 135-136, 82-91.

[33] Zhou, S., \& Peukert, W. (2008). Modeling adhesion forces between deformable bodies by FEM and Hamaker summation. Langmuir, 24, 1459-1468.

[34] Ziskind, G. (2006). Particle resuspension from surfaces: revisited and re-evaluated. Reviews of Chemical Engineering, 22, 1-123. 